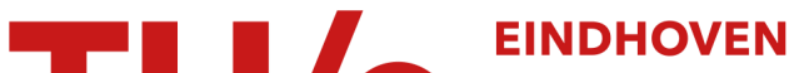 UNIVERSITY OF TECHNOLOGY
}

\section{Optimization of structural and acoustical parameters of bells}

\section{Citation for published version (APA):}

Schoofs, A. J. G., Kroon, P. J. M., \& Campen, van, D. H. (1994). Optimization of structural and acoustical parameters of bells. In A collection of technical papers of the 5th AIAA/USAF/NASA/ISSMO symposium on multidisciplinary analysis and optimization, September 7-9, 1994, Panama City Beach, Fla. Part 2 (pp. 11671180). American Institute of Aeronautics and Astronautics Inc. (AIAA).

Document status and date:

Published: 01/01/1994

\section{Document Version:}

Publisher's PDF, also known as Version of Record (includes final page, issue and volume numbers)

\section{Please check the document version of this publication:}

- A submitted manuscript is the version of the article upon submission and before peer-review. There can be important differences between the submitted version and the official published version of record. People interested in the research are advised to contact the author for the final version of the publication, or visit the DOI to the publisher's website.

- The final author version and the galley proof are versions of the publication after peer review.

- The final published version features the final layout of the paper including the volume, issue and page numbers.

Link to publication

\section{General rights}

Copyright and moral rights for the publications made accessible in the public portal are retained by the authors and/or other copyright owners and it is a condition of accessing publications that users recognise and abide by the legal requirements associated with these rights.

- Users may download and print one copy of any publication from the public portal for the purpose of private study or research.

- You may not further distribute the material or use it for any profit-making activity or commercial gain

- You may freely distribute the URL identifying the publication in the public portal.

If the publication is distributed under the terms of Article $25 \mathrm{fa}$ of the Dutch Copyright Act, indicated by the "Taverne" license above, please follow below link for the End User Agreement:

www.tue.nl/taverne

Take down policy

If you believe that this document breaches copyright please contact us at:

openaccess@tue.nl

providing details and we will investigate your claim. 


\title{
OPTIMIZATION 'OF STRUCTURAL AND ACOUSTICAL PARAMETERS OF BELLS
}

\author{
A.J.G. Schoofs*, P.J.M. Roozen-Kroon**, D.H. van Campen* \\ * Eindhoven University of Technology, The Netherlands \\ ** Fokker Space and Systems, Leiden, The Netherlands
}

\begin{abstract}
The sound spectrum of a bell is the audible sound that is radiated by the vibrating bell, and consists of the superposition of a large number of pure tones, the so-called partials. A partial is the sound field that is radiated by a structural eigenmode of the bell. Using numerical shape optimization a new type of bell was developed in 1985. In this bell the traditional minor-third chord, formed by the lowest partials, was replaced by a major-third chord. Carillons were built using the new bell type. However, perceptional evaluation of the new carillons showed that optimization of the frequencies of the partials only is not enough to ensure a qualitatively good sound spectrum. It is necessary to incorporate other characteristics of the sound spectrum in the optimization process as well. The subject of the present research is the modelling of more characteristics of the sound spectrum, such as the initial strength and the decay rate of the individual partials, and to include these parameters, together with the frequencies, into the optimization process.
\end{abstract}

\section{Introduction}

Traditional North European bells.

North European bells are usually operated either as swinging bells or as carillon bells. In the first case both bell and the clapper form a free swinging pendulum. Carillon bells are fixed mounted and only the clapper is moved by the traction system connected to the keyboard. For both bell applications, traditionally minor-third bells are used. Fig. 1 shows the vertical cross section, the so-called bell profile, including the usual nomenclature.

After a bell is excited with a stroke of the clapper the bell starts to vibrate. The vibration of the bell consists of the superposition of an infinite number of excited eigenmodes with distinct eigenfrequencies. Every eigenmode vibrates with its own frequency in a unique mode shape, has an initial amplitude that is determined by the initial conditions, excitation process and excitation point, and decays with its own decay rate due to the damping related to the eigenmode.

For axi-symmetric bells the eigenmodes can be characterized by the vibrational patterns in the horizontal and vertical cross-sections, shown in Fig. 2. The dotted lines display the undeformed shape of the midplane of the bell.

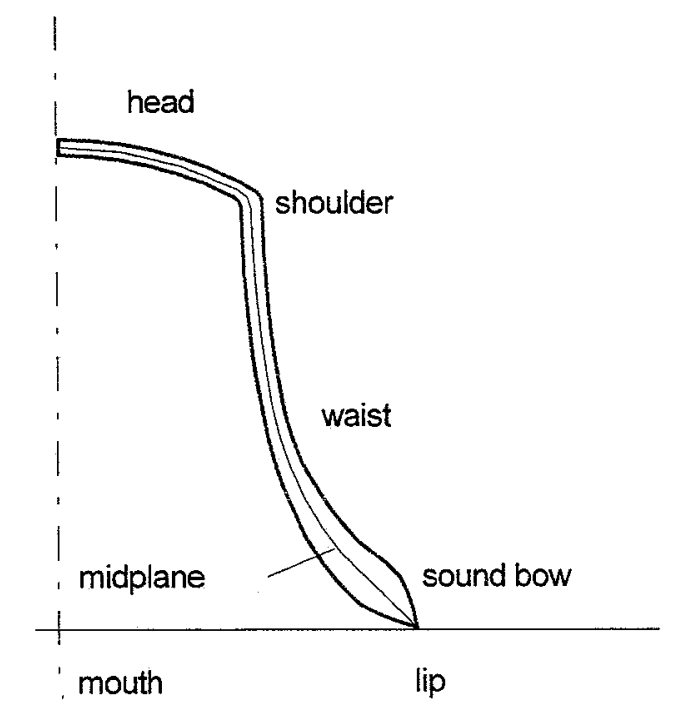

Fig. 1 Bell profile of traditional North European minorthird bell

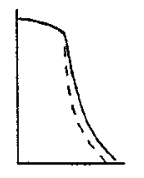

H

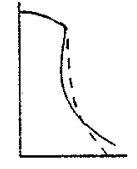

F

vertical vibration patterns
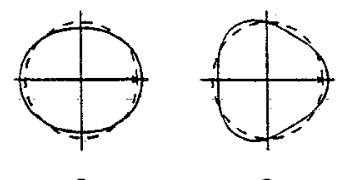

3

2
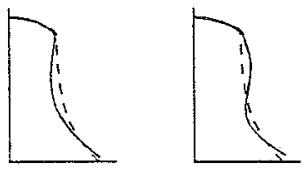

III

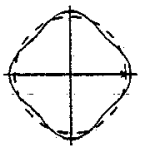

4

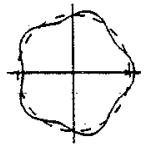

5

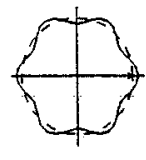

6
Fig. 2 Vibrational patterns of the horizontal and vertical cross-sections of a bell.

Every eigenmode is characterized by its typical vibrational pattern in the horizontal and vertical cross- 
section. The vibrational pattern in the horizontal crosssection is denoted by the numbers $2,3,4,5$, etc.. These numbers indicate the number of periods in circumferential direction (see Fig. 2). The vibrational pattern of an eigenmode in the vertical cross-section is denoted by the roman numbers I, II, III, etc.. The eigenmodes with $\mathrm{m}$ periods in circumferential direction, indicated by the codes I-m II-m, III-m, IV-m, etc., have monotonically increasing eigenfrequencies. There are two exceptions to the vertical vibrational patterns. Instead of the eigenmodes I-2 and II-2, the eigenmodes $\mathrm{H}-2$ and F-2 are found (see Fig. 2).

Every eigenmode excites the air surrounding the bell, generating a vibration in the air with the same frequency. This vibration propagates through the air (away from the bell) generating a sound field. In every point of the sound field the pure tone or partial can be observed.

The sound spectrum radiated by a bell consists of the superposition of a number of partials (i.e. pure tones). Every partial is characterized by its frequency, initial strength or loudness and its decay rate, i.e. the rate with which the strength of a partial diminishes. In Fig. 3 this is illustrated for the seven most important partials of the tradional North European minor-third bell.

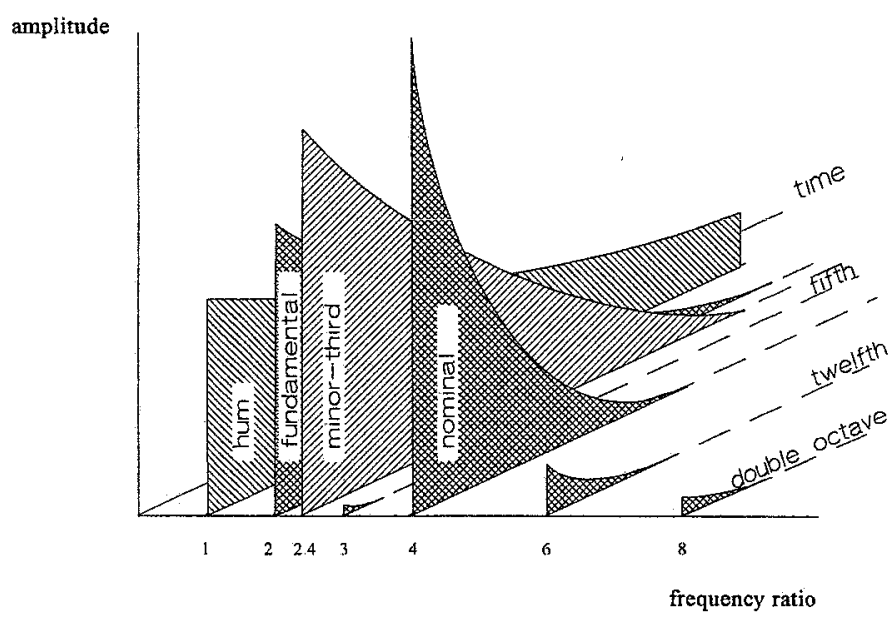

Fig. 3 Schematized representation of the sound spectrum of a traditional North European minor-third bell, showing only the seven most important partials.

For those partials, the frequency ratios and eigenmode codes are given in Table 1 . A frequency ratio 2 (indicating an octave interval) denotes a frequency that is twice as high as the reference frequency. (In Table 1. the hum is the reference partial.) In western music the octave interval is divided in 12 (logarithmically) equal steps, called semitones. The second, third and fourth partial of a minor-third bell form the characteristic minor-third chord in the overtone structure.
Major-third bell design.

The search for the profile of a genuine major-third bell (i.e. a bell whose lowest five partials form a major-third chord, see Table 1.), using numerical shape optimization techniques, was initiated around 1982 by Lehr, the bellfounder of the Royal Eijsbouts bellfoundry in Asten, the Netherlands.

Van Asperen ${ }^{1}$ coupled the FE-analysis of the eigenfrequencies of axi-symmetric structures to an optimization algorithm in the FE-software program DYNOPT. The design variables, used in the optimization, describe the geometry of the axi-symmetric structures to be optimized, e.g. bells. The derivatives of the eigenfrequencies with respect to the design variables, required in the optimization algorithm, are calculated semianalytically.

\begin{tabular}{|l|c|cl|cl|}
\hline partial name & $\begin{array}{c}\text { eigenmode } \\
\text { code }\end{array}$ & \multicolumn{2}{|l|}{$\begin{array}{l}\text { minor-third } \\
\text { freq. } \\
\text { ratio }\end{array}$} & note & \multicolumn{2}{l|}{$\begin{array}{l}\text { major-third } \\
\text { freq. } \\
\text { ratio }\end{array}$} & note \\
\hline hum & $\mathrm{H}-2$ & 1 & $\mathrm{C}_{1}$ & 1 & $\mathrm{C}_{1}$ \\
fundamental & $\mathrm{F}-2$ & 2 & $\mathrm{C}_{2}$ & 2 & $\mathrm{C}_{2}$ \\
third & $\mathrm{I}-3$ & 2.4 & $\mathrm{~Eb}_{2}$ & 2.5 & $\mathrm{E}_{2}$ \\
fifth & $\mathrm{II}-3$ & 3 & $\mathrm{G}_{2}$ & 3 & $\mathrm{G}_{2}$ \\
octave & $\mathrm{I}-4$ & 4 & $\mathrm{C}_{3}$ & 4 & $\mathrm{C}_{3}$ \\
twelfth & $\mathrm{I}-5$ & 6 & $\mathrm{G}_{3}$ & 6 & $\mathrm{G}_{3}$ \\
double octave & $\mathrm{I}-6$ & 8 & $\mathrm{C}_{4}$ & 8 & $\mathrm{C}_{4}$ \\
\hline
\end{tabular}

Table 1. The names of the most important partials, the code of the corresponding eigenmodes, and the frequency ratios of the partials for a traditional minor-third bell and a major-third bell, plus the corresponding notes (based on $\mathrm{C}_{1}$ as hum note).

In $1985 \mathrm{Maas}^{2}$ developed and implemented an efficient model for the accurate description of the bell profile, using a limited number of design variables. He used DYNOPT to optimize the frequency ratios of the five tuneable partials towards the target major-third frequency ratios. Several slightly different bell profiles with nearly ideal overtone structure were calculated. The most promising bell was cast and could be tuned to a genuine major-third bell. (See also Schoofs et al. ${ }^{3}$ and Lehr ${ }^{4}$ ) A complete movable carillon was designed and casted, using the major-third bell shape.

Houtsma and Tholen ${ }^{5}$ investigated the preference of carillonneurs, music students and non-musicians to a minor-third or a major-third carillon. They used a strophe of music, played once in a minor key and once in a major key on a synthesized minor-third and major-third carillon. They found that most carillonneurs preferred the minorthird carillon whatever key the music was in. The music 
students preferred the minor-third carillon for the minorthird strophe of music, and the major-third carillon for the strophe in major key. Most non-musicians preferred the major-third carillon whatever key the music was played in. In agreement with the results from this synthetic perceptual evaluation, field observations have shown that the majorthird carillon is generally appreciated (Lehr $\left.{ }^{6}\right)$. Already several major-third carillons have been installed all over the world.

\section{Objectives of the present research}

The first type major-third bell nevertheless proves to have a noticeably different timbre compared with the minorthird bell. Substantially changing the timbre with the aid of the empirical design rules is impossible. Moreover, the major-third bell has an increased acoustical damping, and is difficult to excite to the same sound power level as the traditional minor-third bell. Higher damping and lower sound power level lead to a considerably shorter sound radiation, coupled with a different timbre of the bell sound. These differences between minor-third bells and the prototype major-third bell clearly lead to the conclusion that the optimization of the frequencies alone is not enough to automatically ensure a generally good-sounding bell. In order to obtain a good bell sound more characteristics of the sound spectrum have to be included in the optimization process.

Therefore, the subject of the present project is the modelling and optimization of the sound spectrum of bells. More specific, the frequency (ratios), strength (ratios) and decay rate (ratios) of the limited number of important partials in the sound spectrum, are modelled and optimized with respect to the geometry of the bell and the clapper.

Next chapter describes the modelling and experimental measuring and/or validation of the important sound spectrum characteristics. In Chapter 3 approximation models of modal eigenfrequencies and modal acoustical damping parameters are described. These models are used in a specially developed multi-staged optimization procedure, and applied to the design of major -third bells (Chapters 4 and 5). Already three different, and interesting types of major-third bells have been designed. Finally in Chapter 6 some conclusions are given.

\section{Modelling and experiments}

This chapter describes the computation of the eigenfrequencies of the bell, the modelling and experimental validation of the collision between bell and clapper, the computation of the acoustical damping parameters, and finally, the measurements of the material and acoustical damping parameters.

\section{Eigenfrequencies}

The geometry and the transient structural dynamic response of a FEM-model of the bell are described using cylindrical co-ordinates, see Fig. 4. A bell is an axi-symmetric structure, subject to non-axi-symmetric loading and deformations, and can be modelled using 8-noded quadrilateral Fourier elements. Only the bell profile in the $r-z$ plane has to be dicretized. In the circumferential direction ( $\varphi$-direction) the displacements and loading of the bell are described by a Fourier series, i.e. the (3np*1)column matrix of the $r-, \varphi$, and $z$-displacements of the np nodal points in the FEM-model of the bell, can be written as:

$$
\left[\begin{array}{l}
\underline{q}_{r}(t, \varphi) \\
\underline{q}_{\varphi}(t, \varphi) \\
\underline{q}_{z}(t, \varphi)
\end{array}\right]=\sum_{m=0}^{\infty}\left[\begin{array}{ll}
\underline{q}_{r m}^{s}(t) \cos m \varphi & \underline{q}_{r m}^{a}(t) \sin m \varphi \\
\underline{q}_{\varphi m}^{s}(t) \sin m \varphi+\underline{q}_{\varphi m}^{a}(t) \cos m \varphi \\
\underline{q}_{z m}^{s}(t) \cos m \varphi & \underline{q}_{z m}^{a}(t) \sin m \varphi
\end{array}\right]
$$

where $m$ denotes the number of periods in circumferential direction. For simplicity of notation, the displacements in the $r-, \varphi$, and $z$-direction are grouped together. The superscripts ' $s$ ' and ' $a$ ' denote symmetric and antisymmetric displacements with respect to the plane $\varphi=0$, respectively.

\section{z-axis}

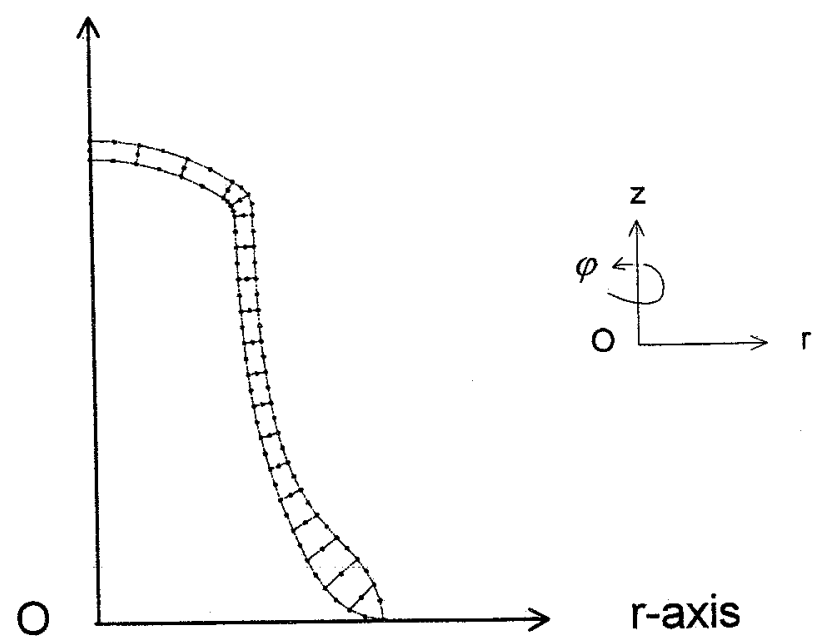

Fig. 4 The FEM-mesh of the bell profile.

Furthermore, if we assume that the bell is to be struck perpendicularly to the $\varphi$-direction, one can choose the plane of the contact point coinciding with the plane $\varphi=0$. Thus, all displacements are symmetric with respect to the plane $\varphi=0$, and can be described by a symmetric Fourier series: 


$$
\underline{q}(t, \varphi)=\left[\begin{array}{l}
\underline{q}_{r}(t, \varphi) \\
\underline{q}_{\varphi}(t, \varphi) \\
\underline{q}_{z}(t, \varphi)
\end{array}\right]=\sum_{m=0}^{\infty}\left[\begin{array}{l}
\underline{q}_{r m}(t) \cos m \varphi \\
\underline{q}_{\varphi m}(t) \sin m \varphi \\
\underline{q}_{z m}(t) \cos m \varphi
\end{array}\right]
$$

The column of symmetric displacement components is abbreviated to:

$$
\underline{q}_{m}(t)=\left[\begin{array}{c}
\underline{q}_{r m}(t) \\
\underline{q}_{\varphi m}(t) \\
\underline{q}_{z m}(t)
\end{array}\right] \quad m=0, \ldots \infty
$$

Applying appropriate kinematic boundary conditions results in the vector of unknown freedoms $\underline{q}_{l m}(t, \varphi)$, for which we substitute:

$$
\underline{q}_{l m}(t, \varphi)=\underline{u}^{*} e^{j \omega t}
$$

where $\omega$ is the eigenfrequency (in $\mathrm{rad} / \mathrm{s}$ ) and $\underline{u}$ the eigenvector, which can be calculated by solving the eigenvalue problem:

$$
\left(-\omega^{2} M_{l l m}+K_{l l m}\right) \underline{u}=\underline{0}
$$

where $M_{l l m}$ is the mass matrix and $K_{l l m}$ is the stiffness matrix related to the unknown displacements, and to the Fourier mode $m$.

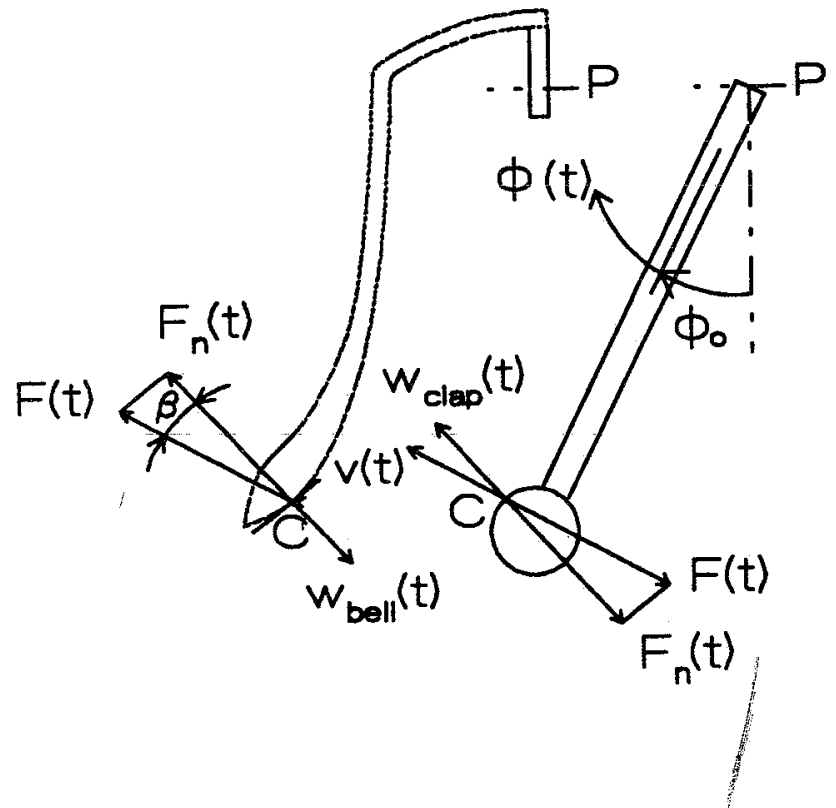

Fig. 5 Forces acting on bell and clapper in contact point $C$

\section{Contact between bell and clapper.}

The contact force $F(t)$ acts perpendicularly to the line through the pivot point $\mathrm{P}$ and the contact point $\mathrm{C}$ on the clapper, see Fig. 5. Generally the contact force $w_{b}(t)$ does not act perpendicularly to the bell wall in the contact point $C$. The force can be decomposed into a normal component $F_{n}(t)$, a tangential component $F_{t}(t)$ (see Fig. 5) and a circumferential component $F_{\varphi}(t)$. The circumferential component $F_{\varphi}(t)$ is zero due to the symmetrical excitation in the $\varphi$-direction.

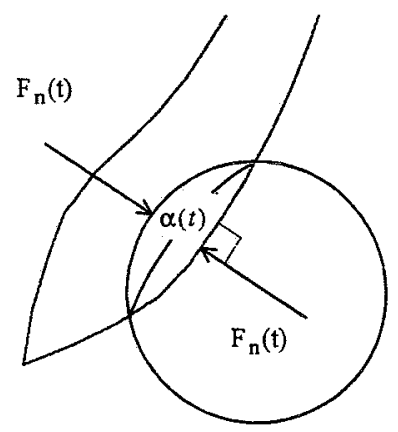

Fig. 6 Local compression between bell and clapper.

The compression $\alpha(t)$ between the bell and the clapper in the contact point (see Fig. 6), perpendicular to the bell wall, is assumed to be caused by the normal component $F_{n}(t)$ of the contact force only. Further it is assumed that the relation between $F_{n}(t)$ and the compression $\alpha(t)$ can - be described by Hertz's law':

$$
F_{n}(t)=k\{\alpha(t)\}^{1.5}
$$

where $k$ is a constant depending on the geometry and the material properties of the colliding bodies. The compression $\alpha(t)$ perpendicular to the bell wall in the contact point $\mathrm{C}$ is defined as the difference between the displacement $w_{c l}(t)$ of the clapper and the displacement $w_{b}(t)$ of the bell, both normal to the bell wall in the contact point (see Fig. 5). The normal displacements $w_{c l}(t)$ and $w_{b}(t)$ will be expressed in the unknown contact force $F(t)$ in order to solve the problem.

The normal displacement $w_{c l}(t)$ of the clapper. The clapper is assumed to be rigid. The displacement $w_{c l}(t)$ is determined from the rotation $\phi(t)$ of the clapper around pivot point $P$ during the collision (see Fig. 5):

$$
w_{c l}(t)=\cos (\beta) l \phi(t)
$$

The rotation $\phi(t)$ during the collision can be determined analytically from the equation of motion of the clapper, linearized around the position $\phi_{0}$ (see Fig. 5): 


$$
\frac{\mathrm{d}^{2} \phi(t)}{\mathrm{d} t^{2}}+\mathrm{A}^{2} \phi(t)=\mathrm{B}-\mathrm{D} F(t)
$$

with $\mathrm{A}^{2}, \mathrm{~B}$ and $\mathrm{D}$ known constants. Thus the displacements $w_{c l}(t)$ can be expressed analytically as a function of the unknown contact force $F(t)$.

The normal displacement $w_{b}(t)$ of the bell. The normal displacement $w_{b}(t)$ of the bell in contact point $C$ will be expressed as a function of the unknown contact force $F(t)$. Since the normal displacement $w_{b}(t)$ can not be determined analytically, $w_{b}(t)$ has to be extracted from the dynamic response of a FE model of the bell excited by the unknown contact force $F(t)$.

The co-ordinates of the contact point $C$, the vector $\vec{n}$ perpendicular to the bell wall in $\mathrm{C}$ and the angle $\beta$ between the direction of the contact force $F(t)$ and the vector $\vec{n}$ are determined from the geometry of the bell and the clapper (see Fig. 5).

The next step is to determine the column with the equivalent nodal forces $f(t, \varphi)$ on the FE model of the bell due to the contact force $F(t)$, as an explicit function of this contact force. Because axi-symmetric Fourier elements are used to model the bell, the nodal forces have to be expressed in a Fourier series in the circumferential direction (i.e. the $\varphi$-direction) A symmetric distribution of the contact force $F(t)$ over the inner bell wall is chosen, and the equivalent nodal forces $\underline{f}(t, \varphi)$ are written as a Fourier series:

$$
\underline{f}(t, \varphi)=\sum_{m=0}^{\infty} \underline{f}_{m}(t) \cos m \varphi
$$

where each Fourier coefficient $\underline{f}_{m}(t)$ is a linear expression in $F(t)$.

The symmetric displacement columns $\underline{q}_{m}(t)$, (see Eq.(3)), caused by the equivalent nodal forces, are calculated from the equations of motion. For each number of periods $m$ in circumferential direction, a separate set of equations of motion has to be solved:

$$
M \ddot{\ddot{q}}_{m}(t)+B_{m} \underline{\dot{q}}_{m}(t)+K_{m} \underline{q}_{m}(t)=\underline{f}_{m}(t), \quad m=0, \ldots \infty
$$

where the matrices $M, B_{m}$ and $K_{m}$ denote the mass, damping and stiffness matrices. Due to the boundary conditions some of the components of $\underline{q}_{m}(t)$ are known. The equations of motion are solved only for the unknown degrees of freedom (DOF). The columns $\underline{q}_{m}(t)$ are written modally using the mass normalized eigenmodes $\underline{X}_{m}^{i}$ of the bell with $m$ periods in the circumferential direction

$$
\underline{q}_{m}(t)=\sum_{i=1}^{n_{\text {eigen }}} \underline{X}_{m}^{i} s_{m}^{i}(t) . \quad m=0, \ldots \infty
$$

with $\underline{s}_{m}^{i}(t)$ are the generalized DOF. Using the modal representation of the columns $\underline{q}_{m}(t)$ and assuming the bell to be weakly damped, we obtain the uncoupled equations of motion in the generalized DOF $\underline{s}_{m}^{i}(t)$ :

$$
\begin{aligned}
& \frac{\mathrm{d}^{2} s_{m}^{i}(t)}{\mathrm{d} t^{2}}+2 \zeta_{m}^{i} \omega_{m}^{i} \frac{\mathrm{d} s_{m}^{i}(t)}{\mathrm{d} t}+\left(\omega_{m}^{i}\right)^{2} s_{m}^{i}(t)=\left(\underline{X}_{m}^{i}\right)^{T} \underline{f}_{m}, \\
& m=0, \ldots \infty, \quad i=1, \ldots n_{\text {eigen }}
\end{aligned}
$$

From Eq. (11) the generalized DOF $\underline{s}_{m}^{i}(t)$ can be obtained analytically. The expressions for the generalized DOF contain a convolution integral ('Duhamel integral', see e.g. ${ }^{8}$ ) with the unknown contact force appearing linearly in the integrand.

From the dynamic response $\underline{q}(t, \varphi)$ of the bell the displacements of the contact point $\mathrm{C}$ on the bell can be calculated, and from this displacements the displacement normal to the bell wall, $w_{b}(t)$. The normal displacement $w_{b}(t)$ is a linear function of the generalized DOF $\underline{s}_{m}^{i}(t)$, and therefore a function of the unknown contact force $F(t)$.

Calculation of the contact force $F(t)$. The obtained expressions for $w_{c l}(t)$ and $w_{b}(t)$ are substituted in Eq. (6):

$$
F(t) \cos \beta=k\left\{w_{c l}(t)-w_{b}(t)\right\}^{1.5}
$$

Eq. (12) is a non-linear equation in the unknown contact force $F(t)$ and can not be solved analytically. Therefore Eq. (13) is dicretized by describing the contact force $F(t)$ to be piece-wise linear:

$$
\begin{gathered}
F(t)=F((i-1) \Delta t)+\frac{1}{\Delta t}\{F(i \Delta t)-F((i-1) \Delta t)\}\{t-(i-1) \Delta t\} \\
t \in[(i-1) \Delta t, i \Delta t], \quad i=1, \ldots n_{\text {end }}
\end{gathered}
$$

Substituting this linear approximation into Eq. (13), the contact force $F(i \Delta t)$ is solved using the previously calculated forces $F(0)$ through $F((i-1) \Delta t)$. The initial contact force $F(0)$ at the start of the collision between bell and clapper is zero. At the same time the displacements of the clapper and the bell are calculated from Eqs. (8) and (12), respectively. 
Validation of the dynamic response calculation. For the experimental validation of the dynamic response a swinging bell with a lip radius of $0.39 \mathrm{~m}$, and a clapper of $9.1 \mathrm{~kg}$, with an overall length of $0.375 \mathrm{~m}$, and a nominal clapper ball radius of $0.52 \mathrm{~m}$ were available. The crown of the bell was rigidly attached to a beam in a frame. The beam and bell were fixed under an angle such that the vertical hanging clapper just touched the bell wall (see Fig. 7). The clapper was given an initial angle of rotation and reproducibly released with the aid of and electro magnet. The contact velocity, resulting from the release angle, was determined from the law of conservation of energy.

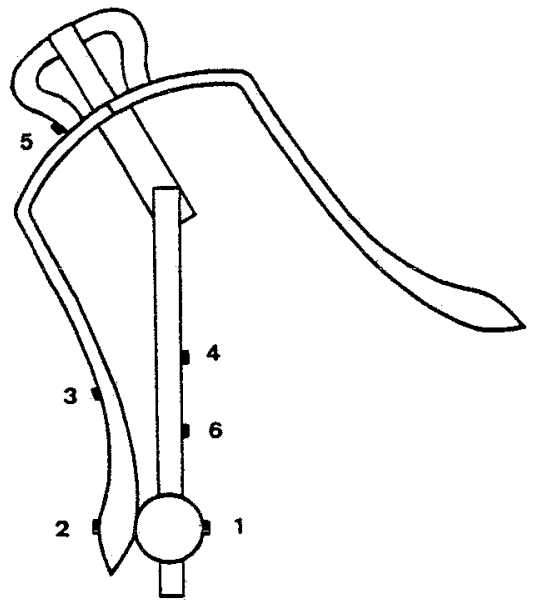

Fig. 7 Configuration of the bell and clapper experiment, and the accelerometer set-up.

The accelerations of the bell wall and the clapper ball were measured on the non-contacting sides of the bell and the clapper, directly opposite to the contact point. Moreover, the accelerations of the clapper rod, and of the crown of the bell were measured. The accelerometers 1 through 6 are indicated in Fig. 7. The simulations of the dynamic response were carried out with a special purpose module of the FEM-program DYNOPT ${ }^{12}$. The accelerations of the clapper ball and the outer bell wall (in the measurement direction) were calculated from the simulated response.

A first comparison of the simulated and the measured accelerations and contact time showed large discrepancies. The simulated accelerations were 30 to $50 \%$ smaller than the measured accelerations, the simulated contact time was some $40 \%$ to large. Sensitivity studies showed that the constant $\mathrm{k}$ in Hertz's law is rather insensitive to changes in the radii of the contact region on the bell, whereas the constant is extremely sensitive to changes in the contact radius of the clapper ball, e.g. because of flattening of the clapper ball. Enlarging the contact radius significantly reduced the contact time, and increased the magnitude of the accelerations of bell and clapper (see Fig. 8 and Fig. 9). Enlarging the contact velocity with $10 \%$ led to an increase of the magnitude of the accelerations of the bell and the clapper, but had little effect on the contact time (see Fig. 8). The rough surface of the clapper ball inhibited the determination of a unique contact radius. Moreover, the contact velocity could not be measure accurately; a deviation of $10 \%$ of the contact velocity was assumed to be realistic.

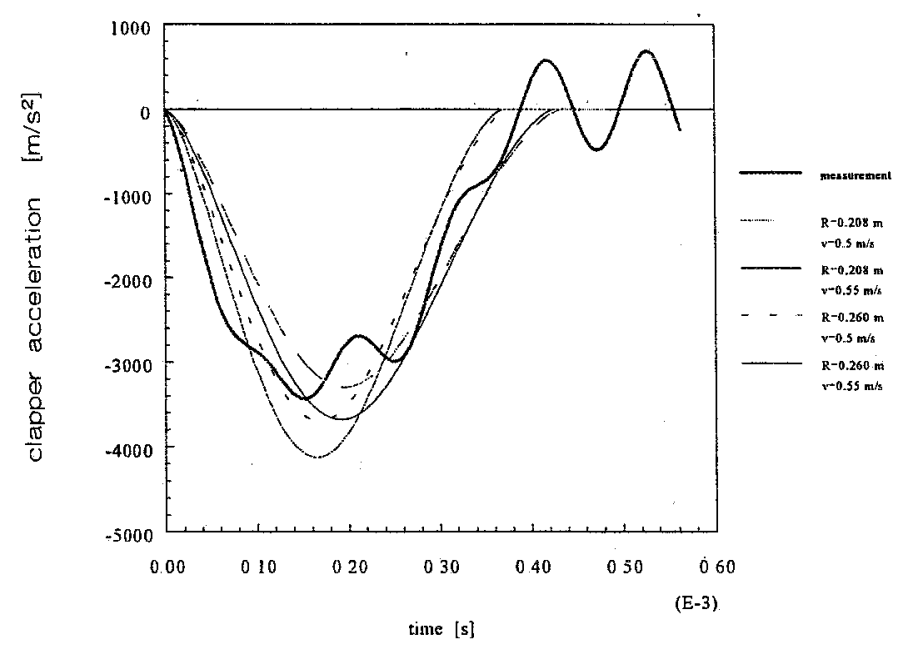

Fig. 8 Measured and simulated clapper ball accelerations.

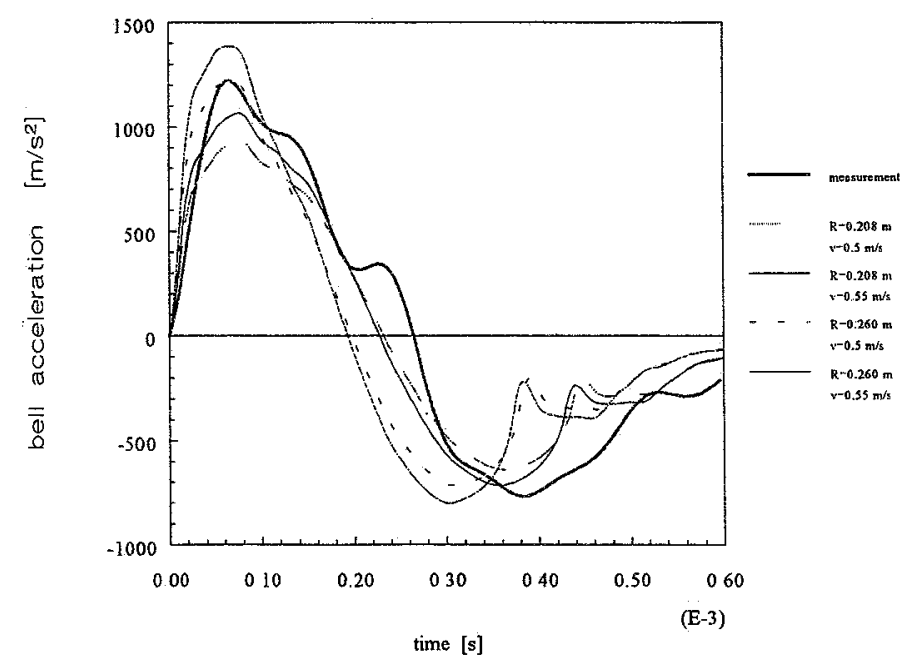

Fig. 9 Measured and simulated bell wall accelerations.

Simulations were carried out, alternatively at $100 \%$ and $110 \%$ of the nominal contact velocity. The radius of the clapper was gradually increased during the simulations, until the simulated and the measured contact times matched. From the clapper ball accelerations in Fig. 8 it can be seen that a contact radius on the clapper ball between $0.208 \mathrm{~m}$ and $0.260 \mathrm{~m}$ ( 4 to 5 times the nominal clapper ball radius) yields simulated contact times that compare very well with the experiment. From the bell wall 
accelerations in Fig. 9 it is concluded that a radius of 0.208 $m$ yields the best results for the contact time.

The contact velocity is found to have a strong influence on the magnitude of the accelerations, whereas the contact time is hardly influenced by a $10 \%$ variation of the contact velocity. It can be seen that the clapper ball acceleration at $0.55 \mathrm{~m}$ contact velocity $(110 \%$ of the nominal velocity) agrees fairly well with the measurements (see Fig. 8), whereas the bell wall accelerations are about $15 \%$ lower than the measured response (see Fig. 9). However, the clapper may not be modelled as a rigid pendulum. The flexibility of the clapper rod superimposes a frequency component of about $10 \mathrm{kHz}$ on the accelerations during the collision (see figures 8 and 9).

Calculation of the acoustical damping using the Boundary Element Method

The modal acoustical damping of a structural eigenmode of the bell is defined as the loss of vibrational energy due to sound radiation into the air surrounding the bell. It is expressed as a fraction of the critical damping, where $\eta=1.0$ corresponds to critical damping. The value of the modal acoustical damping $\eta^{a}$ is calculated from the sound power $P_{s}$ radiated by the eigenmode of the bell:

$$
\eta^{a}=P_{s} / 2 \omega E_{s}
$$

where $\omega$ is the undamped angular eigenfrequency, and $E_{s}$ is the vibrational energy of the eigenmode. Both the angular eigenfrequency $\omega$ and the corresponding eigenmode are obtained, by solving the eigenvalue problem of the bell (using the FEM-program DYNOPT ${ }^{12}$ ). The vibrational energy $E_{s}$, contained in the eigenmode with eigenfrequency $\omega$, is calculated from the bell geometry, the eigenfrequency, the amplitude, and the mode shape of the eigenmode. The radiated sound power $P_{s}$ is determined from the sound pressure distribution and the velocity distribution in the air:

$$
P_{s}=\frac{1}{2} \int_{A} \operatorname{Re}\left(p^{*} v_{n}\right) \mathrm{d} A
$$

where $p^{*}$ is the complex conjugate of the sound pressure $\mathrm{p}, v_{n}$ the particle velocity normal to surface $A$, and $A$ an arbitrary surface encircling the complete bell $\left(\right.$ Fahy $\left.{ }^{3}\right)$. For convenience the surface of the bell can be taken for $A$.

The problem that remains to be solved is the pressure distribution on the bell surface generated by the vibration of the bell. The presence of the air does not affect the eigenfrequency and the mode shape of the eigenmode, due to the minimal fluid loading effects of the air on the bell wall. The only substantial effect of the fluid-structure interaction is the radiation of sound into the air, thereby damping the eigenmode. Ignoring the fluid-structure interaction, the structural and acoustical analysis of the bell can be carried out separately (i.e. uncoupled). To determine the power radiated by an eigenmode it is sufficient to analyse the pressure distribution in the air surrounding the bell, caused by the harmonically vibrating bell wall.

The radiation of sound into the air surrounding the bell is governed by the linearized lossless wave equation describing the propagation of pressure in fluids (Kinsler et $\mathrm{al}^{7}$ ):

$$
\nabla^{2} p(\underline{x}, t)+\frac{1}{c^{2}} p(\underline{x}, t)=0
$$

where $p(\underline{x}, t)$ represents the acoustical pressure, $\underline{x}$ is the column of spatial co-ordinates, $t$ denotes the time, and $c$ is the wave speed of the acoustical waves in the fluid (i.e. air). Eq.(17) is valid for a fluid without internal sources. The excitation of the air by the vibrating bell can be accounted for by the boundary conditions. In the case of the vibrating bell, this is the normal velocity distribution at the surface of the bell. The harmonically vibrating bell excites a harmonically varying sound pressure $p(\underline{x}, t)$ with amplitude $p(\underline{x})$ :

$$
p(\underline{x}, t)=p(\underline{x}) \mathrm{e}^{\mathrm{i} \omega t}
$$

Substituting Eq. (18) into Eq. (17) the wave equation is reduced to the Helmholtz equation:

$\nabla^{2} p(\underline{x})+\mathrm{k}^{2} p(\underline{x})=0$

where the wave number $\mathrm{k}$ is defined as $\mathrm{k}=\frac{\omega}{\mathrm{c}}$. Given the angular frequency $\omega$, and the normal velocity distribution at the boundary, the stationary pressure radiation can be solved from the Helmholtz equation in a specified domain.

Since the radiated power has to be determined, it is necessary to calculate the stationary pressure radiation by the bell, undisturbed by any reflections or sources in the domain. In order to prevent any reflections from the boundaries, the domain is chosen infinitely large. In the complex shaped domain (i.e. the air surrounding the bell) the Helmholtz equation cannot be solved analytically. A numerical method will have to be used to solve the problem. Since the domain is infinitely large, it is not possible to model the complete domain with finite elements. However, it is possible to define a sphere around the bell, that is modelled with finite elements. The spherical boundary is then modelled with a special purpose element that prevents reflections. Another option is the use of the Boundary Element Method (BEM), requiring only 
the discretisation of the domain boundaries. Especially the ease of discretizing the domain boundaries led to the choice of the BEM to analyse the pressure radiation.
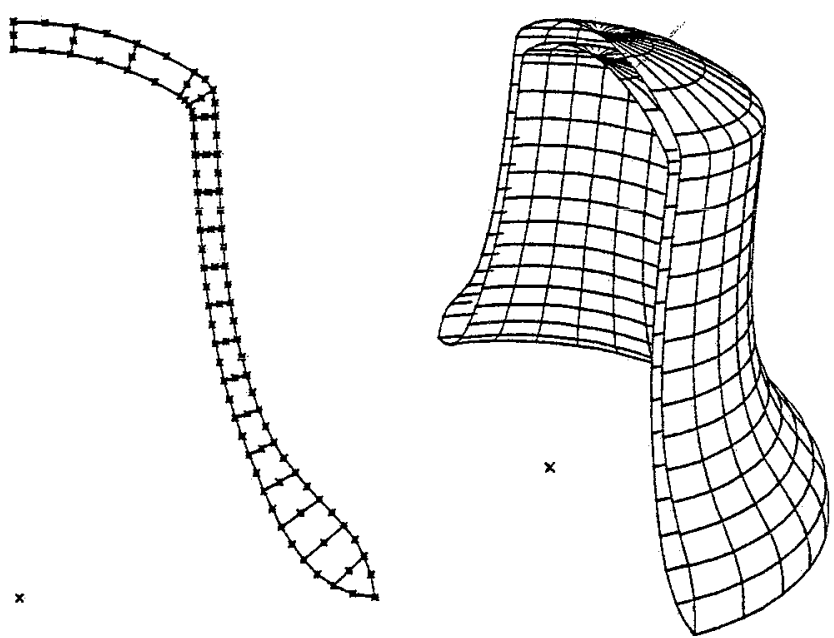

Fig. 10 Two-dimensional FEM-mesh of the bell profile, and the corresponding 3-dimensional BEM- mesh of half the bell.

The undamped angular eigenfrequency and the corresponding mode shape of an eigenmode of a bell can be determined by solving the eigenvalue problem in the FEM-module of DYNOPT, followed by the calculation of the vibrational energy $E_{s}$, using the mass matrix of the bell, the eigenfrequency, amplitude, and the mode shape of the eigenmode. The pressure distribution radiated by an eigenmode was calculated using the Boundary Element Method (BEM) in the acoustical analysis program SYSNOISE $^{16}$. The acoustical analysis program SYSNOISE can solve the Helmholtz equation in Eq. (19) in the frequency domain, given the angular frequency $\omega$, the corresponding normal velocity distribution on the surface of the bell, and a domain on which the equation must be solved. For the analysis of sound radiation into infinity, boundary elements are available in the program SYSNOISE. The surfaces of the inner and outer bell wall were modelled with 8-noded quadratic boundary shell elements.' Since the program SYSNOISE offers no axisymmetric boundary elements with non-axi-symmetric pressure or velocity distribution, it was necessary to model the bell fully in 3 dimensions. Due to existence of planes of symmetry in the eigenmode only a part of the bell has to be modelled. If the mode shape has an even number of periods in circumferential direction, it is sufficient to model a quarter of the bell. For eigenmodes with an uneven number of periods in circumferential direction, half the bell has to be modelled (see Fig. 10). The computational time required for one acoustical analysis is extremely high compared with a FEM-analysis with the same number of degrees of freedom (DOF). This is caused by the fact that the matrices in BEM-analysis are non-symmetric and completely filled, whereas the matrices in a FE-analysis are symmetric and banded.

Measurement of the modal material damping and validation of the modal acoustical damping.

As was mentioned in Chapter 1 the modal damping consists of two parts, the modal material damping and the modal acoustical damping. Material damping values for bell gronze were measured by $\mathrm{Schad}^{2 \mathfrak{Z}}$ and Van Heuven. In order to validate the acoustical damping calculated by means of the BEM, modal damping measurements were carried out (Reinink ${ }^{11}$ ). Measuring the modal damping of an eigenmode, both in plain air and in vacuum, the total modal damping $\eta$ and the modal material $\eta^{m}$, respectively, are obtained. Subtracting the material damping from the total damping, the acoustical damping $\eta^{a}$ is obtained.

The modal material and total damping of a number of eigenmodes of two bells were determined. These bells were minor-third bells with a lip radius of $0.161 \mathrm{~m}$ and $0.174 \mathrm{~m}$. The choice for these relatively small bell was dictated by the size of the compartment, in which the vacuum measurements were carried out. Since it had to be possible to quickly stop the excitation of the bell in the vacuum compartment, a non-contacting excitation method was chosen. Gluing a small iron plate $\left(35^{*} 35^{*} 0.5 \mathrm{~mm}\right)$ to the bell wall in the vicinity of the sound bow, the bell was excited by an electro magnet, that was driven by a sinusoidal current. A sine generator was used to adjust the excitation frequency to the eigenfrequency of a particular eigenmode. The acceleration of the bell wall was measured with an accelerometer. The measurements of the total damping were carried out outside the vacuum compartment.

\begin{tabular}{|l|lll|ll|}
\hline \multirow{3}{*}{ mode } & \multicolumn{3}{|c|}{ experimental } & \multicolumn{2}{c|}{ numerical } \\
\cline { 2 - 6 } & freq. & $\eta^{m}$ & $\eta^{a}$ & freq. & $\eta^{a}$ \\
& {$[\mathrm{~Hz}]$} & {$\left[{ }^{*} 10^{-4}\right]$} & {$\left[{ }^{*} 10^{-4}\right]$} & {$[\mathrm{Hz}]$} & {$\left[{ }^{*} 10^{-4}\right]$} \\
\hline $2-1$ & 801 & 0.952 & 0.515 & 809 & 0.631 \\
$2-2$ & 1525 & 0.780 & 1.132 & 1515 & 0.920 \\
$3-1$ & 1853 & 1.266 & 1.806 & 1842 & 1.466 \\
$4-1$ & 3129 & 2.119 & 0.673 & 3078 & 0.709 \\
$5-1$ & 4642 & 1.497 & 0.757 & 4554 & 0.784 \\
$7-1$ & 8100 & 2.031 & 0.500 & 7956 & 0.401 \\
\hline
\end{tabular}

Table 2. Experimental and numerical damping values and eigenfrequencies for the bell with $\mathrm{R}_{\mathrm{lip}}=0.161 \mathrm{~m}$

In Table 2 the experimental and numerical results for the smallest bell are given. Comparing the experimental and numerical acoustical damping values, it can be concluded that the applied BEM-analysis of the acoustical damping is sufficiently accurate. 


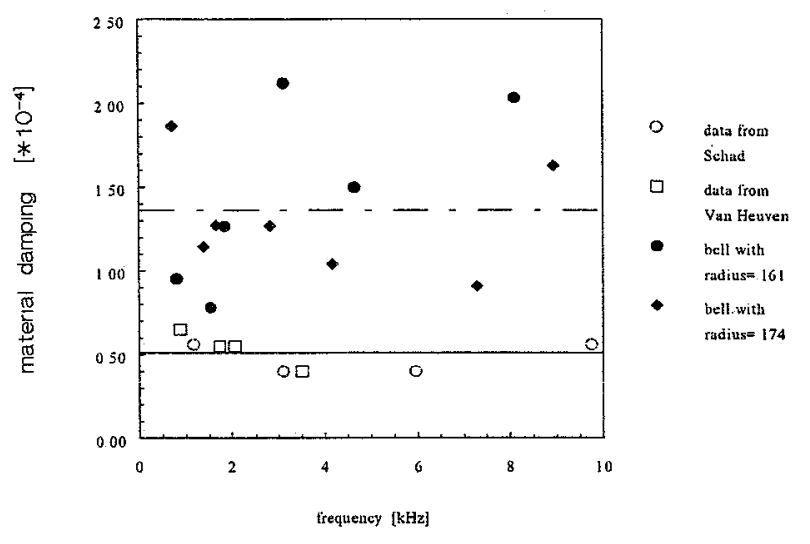

Fig. 11 Experimental modal material damping of the two measured bells, and data given by $\mathrm{Schad}^{13}$ and Van Heuven ${ }^{5}$.

In Fig. 11 the values of the material damping are plotted versus the experimentally determined eigenfrequencies. The material data from Schad and from Van Heuven are given as well. It can readily be seen that the material damping values of the two measured bells are approximately twice as large as the material damping values found by Schad and Van Heuven. This may be caused by a somewhat better quality of the bell bronze used by Schad and Van Heuven, or some additional damping may have been introduced by the supporting frame of the measured bells. Therefore, the mean material damping values obtained by Reinink, Schad and Van Heuven are averaged and the resulting value of $\eta^{m}=0.9^{*} 10^{-4}$ will be used as material damping value in the optimization process.

\section{Approximation models}

In Chapter 2 the modelling of the transient structural dynamic response and the calculation of the related bell sound characteristics have been described. These models will be used as an analysis tool in the optimization of the sound spectrum of bells. However, the analysis of the eigenfrequencies, eigenfrequency derivatives, and eigenmodes of the bell by means of the FEM is rather timeconsuming. This is a serious drawback in optimization, were usually several analyses are required in every iteration step. Even more disastrous is the CPU-time required for the analysis of the modal acoustical damping. The BEManalysis of the acoustical damping of the 7 most important eigenmodes requires about $25 \mathrm{CPU}$-hours on an Alliant FX/40-2 mini supercomputer (or about 45 CPU-minutes on a CRAY Y-MP4/464 supercomputer). The huge CPUtimes required are due to the 3-dimensional BEM-mesh of the bell. Calculation of the acoustical damping derivatives with respect to the bell geometry variables requires an additional 25 CPU-hours for every variable, using finite differences. Obviously, it is almost impossible to incorporate the BEM-analysis of the acoustical damping in the optimization procedure. Therefore, the acoustical damping of the 7 most important partials (listed in Table 1) are modelled with approximation models, that are fitted on numerical data for the acoustical damping, using regression analysis. These regression models for the acoustical damping are explicit functions of the bell geometry variables and the eigenfrequency, and can be easily evaluated.

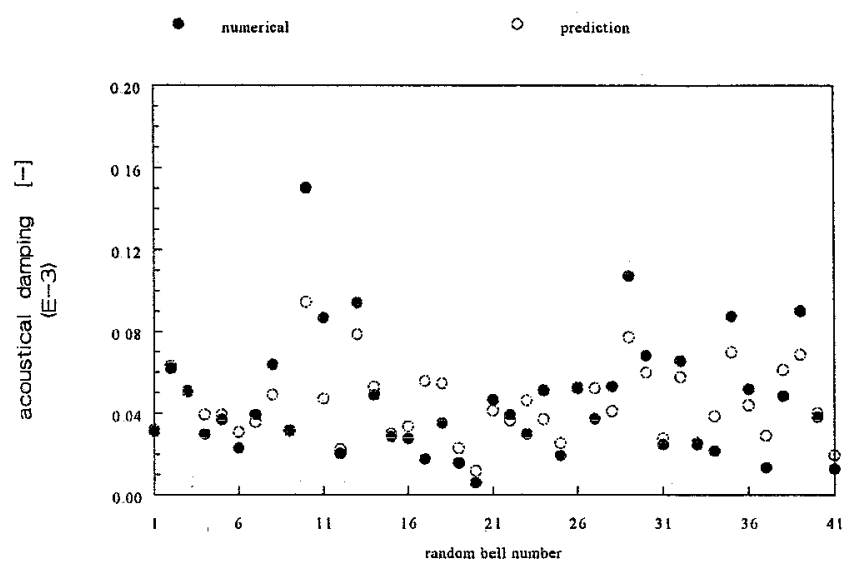

Fig. 12 The BEM-analysis and the prediction with the regression model of the acoustical damping of the hum for the reference bell and 40 bells of randomly chosen geometry.

Fig. 12 illustrates the prediction capabilities of the regression models for the hum. Although for a number of bells the approximation agrees fairly well with the BEManalysis, also several bells can be noticed with rather large deviations between prediction and BEM-analysis. In future research the approximation models for the acoustical damping should be improved in order to provide a more reliable tool to be used in the optimization process.

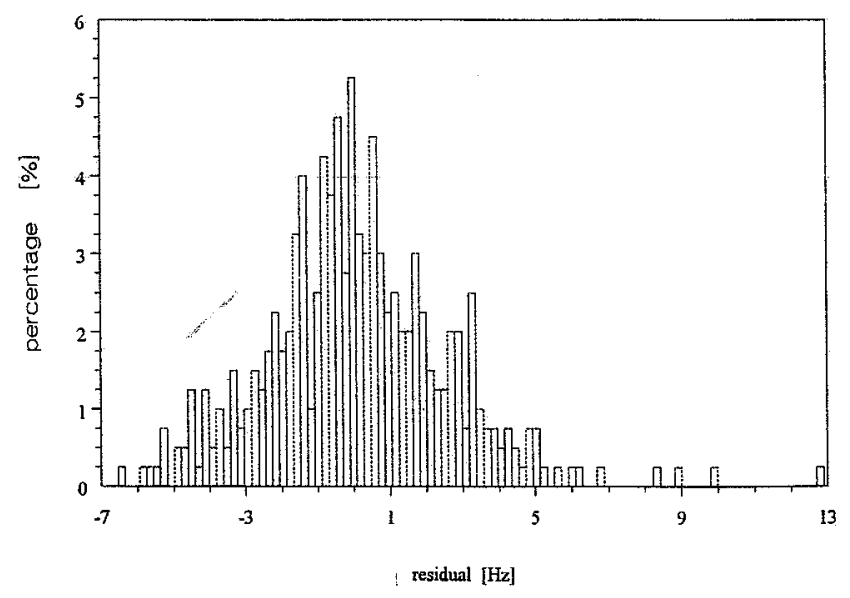

Fig. 13 Distribution of the residuals of FEMeigenfrequencies and predicted frequencies of the hum for 400 randomly chosen bell geometries. 
Furthermore, the option to choose a starting bell geometry for the optimization that nearly satisfies the target values for the most important eigenfrequency ratios (and decay rat ratios), would save many FEM-based optimization iterations and a considerable amount of CPUtime. Therefore, the most important eigenfrequencies (listed in Table 1) are modelled with approximation models as well, fitting the approximation models on numerical data of the eigenfrequencies, by means of regression analysis. In Fig. 13 the residuals of the approximated eigenfrequency of the hum are plotted (Roozen-Kroon ${ }^{12}$ ). The FEMcalculated eigenfrequencies of the test bells are very well predicted; only a few outliers are encountered.

At the time the eigenfrequency approximation models were built, a FEM-analysis of the 7 eigenfrquencies required 15 CPU-minutes on an Apollo D-3000 workstation $\left(\mathrm{Schoofs}^{15}\right)$. Meanwhile the same FEManalysis costs about 2 CPU-minutes on an Alliant FX/40-2 computer. But even now it is worthwhile to use the approximation models of both the eigenfrequencies, and the acoustical damping for a quick and rough localisation of local optima in the bell geometry domain, that can be used as starting bell geometries. Of course, approximation models for the acoustical damping are mandatory at this time.

\section{Optimization}

\section{Bell shape design variables}

Schoofs ${ }^{15}$ developed a method for an accurate bell profile description, using a limited number of bell design variables. In this method the radii of a number of basic points on the midplane of the bell are used as variables describing the geometry of the bell midplane (see Fig. 14).

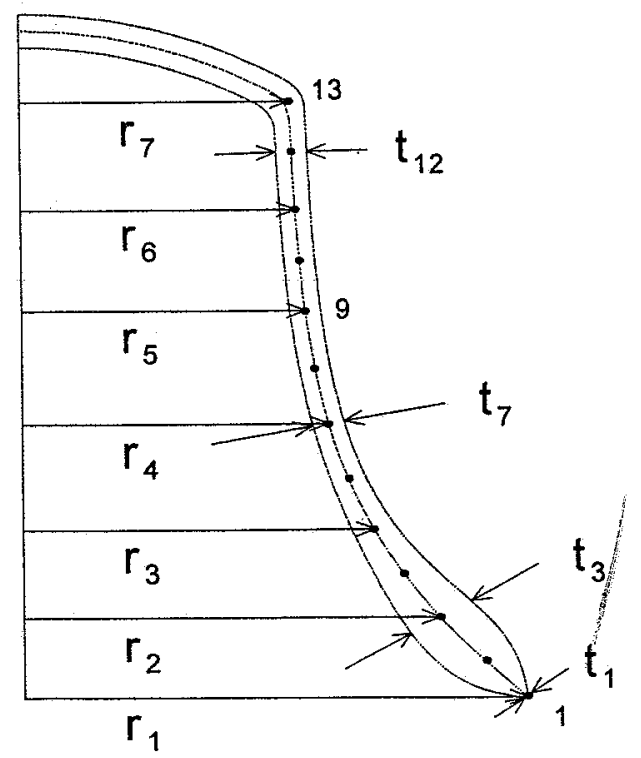

Fig. 14 Bell shape design variables.
These basic points are more or less equally spaced along the midplane of the bell, and define a cubic spline curve. In every basic point the thickness of the bell, measured perpendicularly to the midplane, is given as well. Another cubic spline describes the thickness data, thus defining the inner and outer bell wall. The head and the shoulder of the bell have a predefined shape, that is scaled to match the radius and the thickness of the bell just below the shoulder.

\section{Optimization strategy}

In order to optimize the selected characteristics of the sound spectrum, a "black box" optimization algorithm might be used that optimizes all characteristic at the same time. However this approach has some serious drawbacks. The major disadvantage of a "black box" optimization is the fact that during each iteration all characteristics are optimized with respect to all design variables of the bell and the clapper. But obviously it is useless to optimize the strength ratios with respect to the contact point position if the frequency ratios and decay ratios of the partials, that depend only on the design variables of the bell geometry, are not yet near their target values. Moreover, the computational effort required to optimize everything simultaneously will be much greater than the effort required when optimizing the characteristics in subsequent stages. Another problem using the "black box" optimization is the choice of the target values and the corresponding required accuracy's. It is always possible that the selected combination of target values for frequency ratios and decay rate ratios cannot be reached for some partials, especially when trying to find a bell and clapper geometry matching a completely new sound spectrum. In that case it will be necessary to alter some target values or some accuracy's required. However, this can only be concluded after a large number of iterations. To overcome these disadvantages a multi-staged optimization strategy is used.

The most important characteristics of the sound spectrum are the frequency ratios of the lowest partials. Nowadays, bell founders can correctly tune the lowest five partials of a well cast bell. "Well cast" means that the two more partials, the "twelfth" and the "double octave" (see Table 1), must be acceptable without tuning. The frequency ratios of those seven partials must correspondent to musically well defined intervals, see Table 1 . If the frequency ratios of a bell do not sufficiently approximate the target values, the bell is not tuneable and therefore worthless. Hence, in the optimization of the sound spectrum of a bell the frequency ratios are the most important optimization criteria. The goal of the first stage of the optimization strategy is therefore, to find a bell geometry that satisfies (within the required accuracy's) the target values of the frequency ratios and decay rate ratios. Since the frequency ratios and decay rat ratios both only 
depend on the bell geometry, it is necessary to optimize them simultaneously.

Another important bell characteristic is the so-called $\mathrm{fD}$-parameter. The $\mathrm{fD}$-parameter is defined as the product of the frequency of the hum partial (in $\mathrm{Hz}$ ) and the lip diameter of the bell (in $\mathrm{m}$ ). In traditional North European bells the fD-value lies between 190 and 210 . The $\mathrm{fD}-$ parameter is used as a global measure for the wall thickness of the bell. For practical reasons the wall thickness of the smallest bells in a carillon are increased, to $\mathrm{fD}=400$, or even higher.

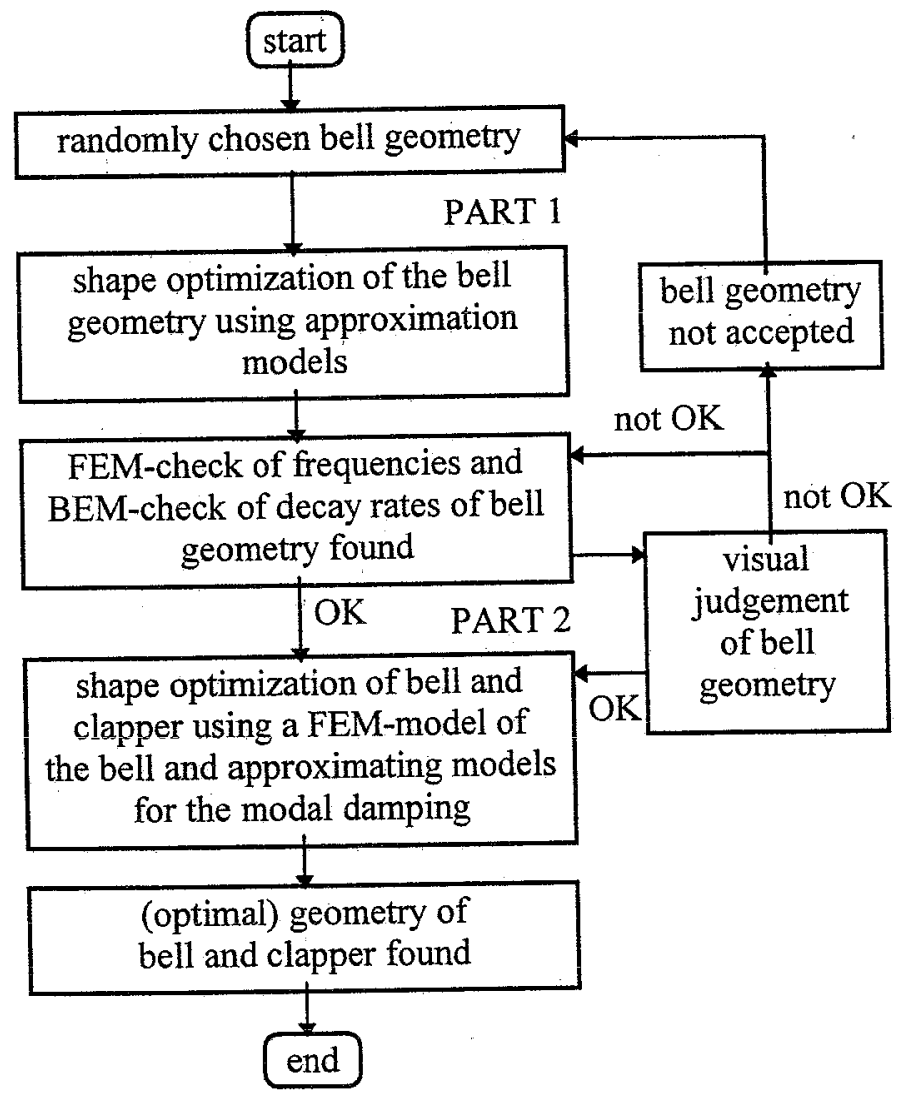

Fig. 15 Flowchart of the optimization process.

The strength ratios of the partials will be optimized in the second stage, keeping the bell geometry resulting from the first stage, constant. In the second stage the strength ratios are optimized with respect to the contact point position only.

Summarizing, the optimization process is divided into two main parts, shown in Fig. 15. In the first part, the frequency ratios, decay rate ratios, and $\mathrm{fD}$-parameter are optimized using approximation models to describe the frequencies, and the modal acoustical damping values as a function of the bell geometry variables. If an FEM and BEM analysis of the resulting bell geometry confirms that the frequency and decay rate ratios are close enough to the target values, and the $\mathrm{fD}$-parameter is near the target value, the geometry is used as a starting bell geometry for the
FEM-analysis based optimization of the bell. In the second part the frequency ratios, decay rate ratios, and $\mathrm{fD}$ parameter, and the partial strength ratios are optimized using a FEM-model to calculate the eigenfrequencies of the bell, instead of the approximation models for the eigenfrequencies. The acoustical damping values are still calculated by means of the approximation models. Using the bell geometry the first stage as a starting bell geometry for the FEM-analysis based optimization in the second part, many FEM-iterations are avoided, resulting in a reduction of the CPU-time required for the optimization of the sound spectrum.

\section{$\underline{\text { 5. Results }}$}

The procedure described in the previous chapter has been applied to the optimization of major third bells

"Fast" optimization of major-third bells.

In part one several optimization runs are carried out, using different starting bell geometries and different sets of weighting factors for the frequency and decay rate components of the objective function. From the resulting solutions a number of bell geometries are selected, for which most of the frequency ratios and decay rate ratios (both calculated by means of the approximation models) are reasonably close to the target values. For these bell geometries the frequency ratios and the acoustical damping values of the seven most important partials are checked by means of the FEM and the BEM, respectively. Thus a number of promising bells have been found, with frequency ratios, total damping values and $\mathrm{fD}$-value that exhibit small to moderate deviations from the target values. These deviations from the target values, however, may sufficiently improve during the FEM-based optimization of the bell geometry. In Table 3 the results of three promising bells are listed. For the target frequency and target damping values, it is referred to Table 4 .

\begin{tabular}{|l|ll|ll|ll|}
\hline \multirow{2}{*}{$\begin{array}{l}\text { partial } \\
\text { code }\end{array}$} & \multicolumn{2}{|c}{ bell 6 } & \multicolumn{2}{c|}{ bell 7 } & \multicolumn{2}{c|}{ bell 8 } \\
freq. & damp. & freq. & damp. & freq. & damp. \\
\hline $2-1$ & 1.000 & 1.130 & 1.000 & 1.217 & 1.000 & 1.128 \\
$2-2$ & 2.055 & 1.572 & 2.021 & 1.679 & 2.085 & 1.636 \\
$3-1$ & 2.528 & 2.025 & 2.481 & 1.951 & 2.504 & 1.947 \\
$3-2$ & 3.048 & 2.628 & 2.970 & 2.729 & 3.030 & 2.757 \\
$4-1$ & 4.157 & 4.034 & 4.129 & 3.622 & 4.155 & 4.159 \\
$5-1$ & 6.100 & 1.698 & 6.075 & 1.792 & 6.077 & 1.679 \\
$6-1$ & 8.351 & 1.823 & 8.261 & 1.633 & 8.262 & 1.975 \\
\hline & fD $=198.0 \mathrm{~m} / \mathrm{s}$ & $\mathrm{fD}=209.8 \mathrm{~m} / \mathrm{s}$ & $\mathrm{fD}=194.4 \mathrm{~m} / \mathrm{s}$ \\
\hline
\end{tabular}

Table 3. Frequency ratios, total damping values, and $\mathrm{fD}$ value of three promising bells, selected after "fast" optimization (frequencies and damping values have been computed by means of FEM and BEM, respectively). 
FEM-based optimization of major third bells.

After the "fast" optimization was carried out, the three promising bell geometries have been used as starting bell geometries in part 2 of the optimization process (see Fig. 15). In this optimization stage the frequencies are calculated by means of the FEM, whereas the acoustical damping values are still calculated by means of the approximation models. A Sequential Linear Programming algorithm is used to solve the optimization problem.

In the previous section it has been concluded that he acoustical damping values cannot be calculated accurately enough by the approximation models for optimization purposes. Assuming that the bell geometry and, in consequence, the acoustical damping will not vary very strongly during the second stage of the optimization, however, the approximation models can be used to fix the acoustical damping at the current values (calculated by the approximation models). Of course, this has to be checked at the end of the FEM-based optimization procedure.

For bell 6 (see Table 3) the lowest five eigenfrequency ratios are easily optimized within about $0.25 \%$ of the target frequency ratios. All frequency ratios of the final geometry of bell 6 are within about $0.5 \%$ of their target values, with exception of the twelfth (partial code 5-1), see Table 4 . The final bell 6 can easily be tuned to the required accuracies of the lowest five partials. Comparing the frequency ratios of the starting bell geometry and the final geometry (see Table 4), it can be concluded that the frequency ratios of the partials 4-1, 5-1 and 6-1 have been decreased substantially. The decrease of the frequency ratios of the lowest four partials is more modest.

\begin{tabular}{|c|c|c|c|c|c|c|}
\hline \multirow[b]{2}{*}{$\begin{array}{l}\text { partial } \\
\text { code }\end{array}$} & \multirow{2}{*}{$\begin{array}{l}\text { target } \\
\text { freq. } \\
\text { ratio }\end{array}$} & \multirow{2}{*}{$\begin{array}{l}\text { target } \\
\text { damp. } \\
{\left[{ }^{*} 10^{-4}\right]}\end{array}$} & \multicolumn{2}{|c|}{ start bell 6} & \multicolumn{2}{|c|}{ final bell 6} \\
\hline & & & $\begin{array}{l}\text { freq. } \\
\text { ratio }\end{array}$ & $\begin{array}{l}\text { damp. } \\
{\left[* 10^{-4}\right]}\end{array}$ & $\begin{array}{l}\text { freq. } \\
\text { ratio }\end{array}$ & $\begin{array}{l}\text { damp. } \\
{\left[* 10^{-4}\right.}\end{array}$ \\
\hline $2-1$ & 1.000 & 1.11 & 1.000 & 1.130 & 1.000 & 1.121 \\
\hline $2-2$ & 2.000 & 1.64 & 2.055 & 1.572 & 2.001 & 1.441 \\
\hline $3-1$ & 2.520 & 1.62 & 2.528 & 2.025 & 2.516 & 1.810 \\
\hline $3-2$ & 2.997 & 3.71 & 3.048 & 2.628 & 3.000 & 2.667 \\
\hline $4-1$ & 4.000 & 3.82 & 4.157 & 4.034 & 4.010 & 3.551 \\
\hline $5-1$ & 5.993 & 1.61 & 6.100 & 1.698 & 5.859 & 1.646 \\
\hline \multirow[t]{2}{*}{$6-1$} & 8.000 & 1.55 & 8.351 & 1.823 & 8.039 & 1.887 \\
\hline & \multicolumn{2}{|c|}{$\mathrm{fD}=200.0 \mathrm{~m} / \mathrm{s}$} & \multicolumn{2}{|c|}{$\overline{\mathrm{fD}}=1 \overline{9} \overline{8} . \overline{0} \mathrm{~m} / \mathrm{s}$} & \multicolumn{2}{|c|}{$\mathrm{fD}=\overline{19} \overline{7} . \overline{\mathrm{m}} / \mathrm{s}$} \\
\hline
\end{tabular}

Table 4. Frequency ratios, total damping values, and $\mathrm{fD}$ value of the starting bell geometry and the final bell geometry of bell 6 (frequencies and damping values are computed by means of FEM and BEM, respectively). The target values are given as well.

The FEM-based optimization has also been carried out for the bells 7 and 8, which could be optimized to the target values within a sufficient accuracy as well. The starting bell geometries and the final geometries of the bells 6,7 , and 8 are visualized in Fig. 16.

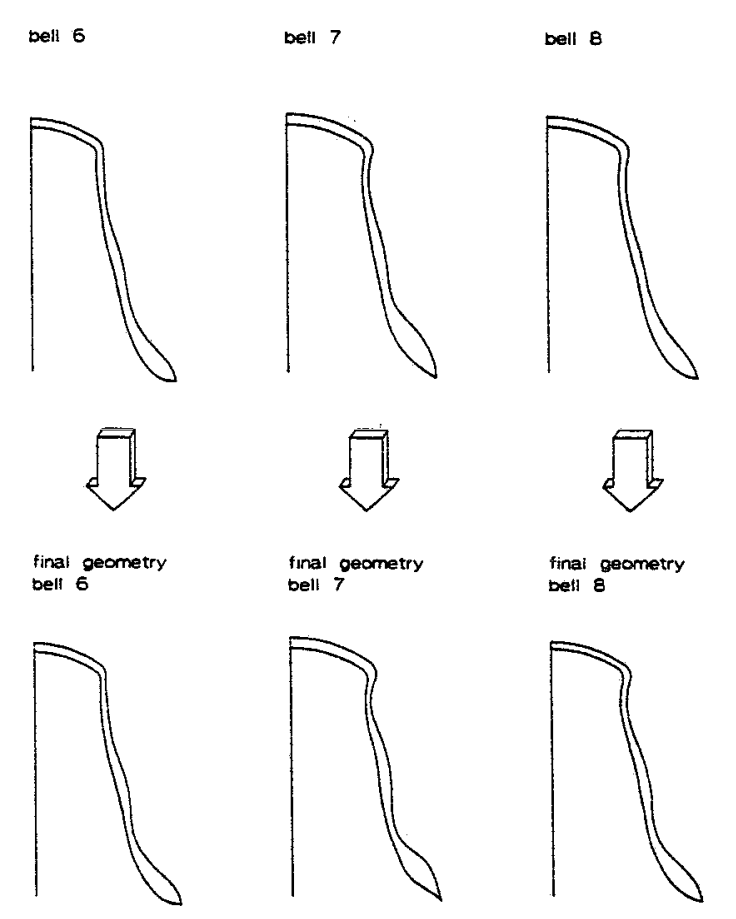

Fig. 16 Starting and final geometries of bells 6,7 , and 8

Optimization of the contact point position. In the previous sections the shape optimization of the bell geometry with respect to the frequency ratios, decay rate ratios, and $\mathrm{fD}$ parameter has been carried out, resulting in the three interesting major $t$-third bell geometries 6,7 and 8 . The last stage in the optimization procedure is the optimization of the contact point position, and the determination of a suitable clapper configuration for these three bells. We have decided to evaluate the radiated sound power ratios of the reference minor-third bell to a medium force excitation, and to use these sound power ratios as target values during the contact point optimization.

\begin{tabular}{|c|c|c|c|c|}
\hline $\begin{array}{c}\text { partial } \\
\text { code }\end{array}$ & $\begin{array}{c}\text { power } \\
\text { ratio } \\
\text { bell 6 } \\
\mathrm{r}_{\mathrm{C}}=0.428 \\
\mathrm{z}_{\mathrm{C}}=0.047\end{array}$ & $\begin{array}{c}\text { power } \\
\text { ratio } \\
\text { bell 7 } \\
\mathrm{r}_{\mathrm{C}}=0.440 \\
\mathrm{z}_{\mathrm{C}}=0.084\end{array}$ & $\begin{array}{c}\text { power } \\
\text { ratio } \\
\text { bell 8 } \\
\mathrm{r}_{\mathrm{C}}=0.438 \\
\mathrm{z}_{\mathrm{C}}=0.068\end{array}$ & $\begin{array}{c}\text { target } \\
\text { power } \\
\text { ratio }\end{array}$ \\
\hline $2-1$ & 1.000 & 1.000 & 1.000 & 1.000 \\
$2-2$ & 1.948 & 1.938 & 1.795 & 2.36 \\
$3-1$ & 13.322 & 10.668 & 12.876 & 9.78 \\
$3-2$ & 0.145 & 0.205 & 0.171 & 0.10 \\
$4-1$ & 42.238 & 43.876 & 42.830 & 42.15 \\
$5-1$ & 8.735 & 10.337 & 9.684 & 7.35 \\
$6-1$ & 4.599 & 4.299 & 5.871 & 2.29 \\
\hline
\end{tabular}

Table 5. Sound power ratios and corresponding optimal contact point position for the bell 6,7 and 8 . The sound power ratios are calculated for the acoustical damping values obtained from the BEM. 
By numerical optimization of the contact point position it has appeared that the sound power ratio of partial 2-2 is always too low, whereas the ratio of partial 31 is always too high. In Table 5 the resulting sound power ratios and the corresponding contact point positions are listed. Moving the contact point along the lower inner bell wall leads to a simultaneous increase or decrease of the partials 2-2 and 3-1, prohibiting a contact point where both target sound power ratios are reached.

\section{Conclusions}

In this paper the modelling and optimization of the sound spectrum characteristics of carillon bells has been described. In the project structural optimization software has been developed for the design of bell geometries with specified sound spectrum characteristics. Essential is the modelling of the sound spectrum characteristics and the underlying modal properties of the transient structural dynamic response of the clapper-excited bell as function of the variables describing the bell and clapper geometry. In this research project modelling and optimization have been carried out for carillon bells, but the method can also be applied to similar axi-symmetric structures.

Eigenfrequencies and eigenmodes are calculated by means of a FEM program, employing axi-symmetric finite elements with non-axi-symmetric deformations. The timedependent amplitudes of the eigenmodes are calculated using Hertz's law to model the contact between the bell and the clapper. The clapper is modelled as a rigid pendulum. The experimental validation of the contact model shows that the dynamic response of the clapperexcited bell is sufficiently accurate, although the flexibility of the clapper clearly influences the bell and clapper accelerations.

The FEM-calculation of the eigenfrequencies of a bell is rather time-consuming, but the CPU-time required for the calculation of the acoustical damping of the seven most important partials of a bell is totally unacceptable during an iteration of the optimization. Therefore the eigenfrequencies and the acoustical damping have been described by regression models, fitted on numerical data obtained by means of the FEM and BEM, respectively. The accuracy of the regression models of the acoustical damping has appeared to be rather poor, although the predicted damping is usually of the correct order of magnitude. The regression models have been very useful in the optimization process.

From the experience with the optimization software it is concluded that it is impossible to automate the optimization of a large number of sound spectrum characteristics. The designer must always judge the intermediate results, rejecting some solutions, or adjusting weighting factors and/or target values, enabled by the developed multi-staged optimization procedure.
Employing the developed software to optimize the major-third bell timbre, it appears that it is extremely difficult to find a bell geometry that fulfils all the target values. In/part this is caused by the inaccuracy of the approximation models of the acoustical damping. Every interesting bell requires a check of the acoustical damping by means of a time-consuming BEM-analysis. For the most part, however, this is caused by the virtual incompatibility of target values. A minor-third bell fulfilling the target values is known to exist, but there is no guarantee that a major-third beli can be found that fulfils most (or all) of the target values. Therefore, the designer has to compromise, judging what target values must be reached, and allowing a number of characteristics not to reach the target values. Hence, the use of the optimization software requires a certain amount of experience and expertise in order to obtain useful bell geometries. Nevertheless, already three interesting major-third bell geometries and suitable contact point positions have been found.

\section{Acknowledgement}

This research has been supported by the Dutch Technology Foundation (STW), grant no. EWT 77.1246.

Computer resources has been provided by the Foundation for National Computer Facilities.

\section{$\underline{\text { References }}$}

1. F.J.G. van Asperen, The optimization of the eigenfrequencies of axi-symmetric structures, applied to swinging bells and carillon bells (in Dutch), Eindhoven University of Technology, graduation thesis, report WFW84.012, 1984.

2. K.-J. Bathe, E.L. Wilson, Numerical methods in finite element analysis, Prentice Hall, London, 1976.

3. F.J. Fahy, Sound and structural vibration: radiation, transmission and response, Academic Press, London, 1985.

4. W. Goldsmith, Impact, the theory and physical behaviour of colliding bodies, Edward Arnold LTD., London, 1960.

5. E.W. van Heuven, Acoustical measurements on churchbells and carillons, Delft University of Technology, PhD-thesis, 1949.

6. A.J.M. Houtsma, H.J.G.M. Tholen, A carillon of majorthird bells II : A perceptual evaluation, Music Perception, vol. 4, no. 3, pp. 255-266, 1987.

7. L.E. Kinsler, A.R. Frey, A.B. Coppens, J.V. Sanders, Fundamentals of acoustics, John Wiley, New York, 1982.

8. A. Lehr, A carillon of major-third bells II : From theory to practice, Music Perception, vol. 4, no. 3, pp. 267280,1987 
9. A. Lehr, The designing of swinging bells and carillon bells the past and present, Athanasius Kircher Foundation, Asten, The Netherlands, 1987.

10. P.J.J. Maas, Investigations of the geometry of a majorthird bell (in Dutch), Eindhoven University of Technology, graduation thesis, report WFW85.027, 1985.

11. H.F. Reinink, Experimental investigation of the damping of bells (in Dutch), Eindhoven Univ. of Techn., report WFW91.110, 1991.

12. P.J.M. Roozen-Kroon, Structural optimization of bells, Eindhoven Univ. of Techn., PhD-thesis, 1992.

13. C.R. Schad, H, Warlimont, Acoustic investigations on the influence of the material on the sound of bells (in German), Acustica, vol. 29, no. 1, pp 1-14, 1973.

14. A. Schoofs, F. van Asperen, P. Maas, A. Lehr, A carillon of major-third bells I : Computation of bell profiles using structural optimization, Music Perception, vol. 4, no.3, pp. 255-266,1987.

15. A.J.G. Schoofs, Experimental design and structural optimization, Eindhoven Univ. of Techn., PhD-thesis, 1987.

16. Sysnoise 4.3, Theoretical manual, publ. by Dynamic Engineering NV, Heverlee, Belgium, 1990. 